\title{
A Stochastic Turbidostat Model With Ornstein- Uhlenbeck Process: Dynamics Analysis and Numerical Simulations
}

Daqing Jiang ( $\sim$ daqingjiang2010@hotmail.com )

China University of Petroleum https://orcid.org/0000-0003-2439-6292

Xiaojie Mu

China University of Petroleum Huadong - Qingdao Campus

Tasawar Hayat

King Abdulaziz University

Ahmed Alsaedi

King Abdulaziz University

\section{Research Article}

Keywords: Stochastic turbidostat model, Ornstein-Uhlenbeck process, Extinction, Stationary distribution

Posted Date: June 30th, 2021

DOI: https://doi.org/10.21203/rs.3.rs-660384/v1

License: (c) This work is licensed under a Creative Commons Attribution 4.0 International License. Read Full License 


\section{Abstract}

Many turbidostat models are affected by environmental noise due to various complicated and uncertain factors, and Ornstein-Uhlenbeck process is a more effective and precise way. We formulate a stochastic turbidostat system incorporating Ornstein-Uhlenbeck process in this paper, develop dynamical behavior for the stochastic model, which include the existence and uniqueness of globally positive equilibrium, sufficient conditions of the extinction, the existence of a unique stationary distribution and an expression of density function of quasi-stationary distribution around the positive solution of the deterministic model. The results indicate that the weaker volatility intensity can

ensure the existence and uniqueness of stationary distribution, and the stronger reversion speed can lead to the extinction of microorganism. The validity of analytical results is verified through numerical simulation, which assess the influence of the reversion speed and the volatility intensity on the long-term behavior of microorganism.

\section{Full Text}

This preprint is available for download as a PDF. 